\title{
Qualidade de vida e a cirurgia em cancro da mama: revisão narrativa da literatura
}

Quality of life and breast cancer surgery: narrative literature review

\section{Calidad de vida y la cirugía en cáncer de mama: revisión narrativa de la literatura}

\author{
Ana Cristina Eberhardt ${ }^{1}$ ORCID Samuel Lincoln Bezerra Lins ${ }^{2}$ ORCID \\ 'Universidade do Porto \\ Portugal
}

Fecha correspondencia:

Recibido: febrero 17 de 2016.

Aceptado: enero 12 de 207.

Forma de citar:

Eberhardt, A.C., \& Lins, S.L.B.

(2017). Qualidade de vida e a

cirurgia em cancro da mama:

revisão narrativa da literatura Rev.

CES Psicol., 10(1), 35-47.

Open access

(c) Copyright

Licencia creative commons

Etica de publicaciones

Revisión por pares

Gestión por Open Journal System

DOl: http://dx.doi.org/10.21615/

cesp.10.1.3

ISSN: 2011-3080

Sobre los autores:

1. Mestre em Oncologia pelo Instituto de Ciências Biomédicas Abel Salazar. Universidade do Porto, Portugal.

Comparte

F日G. $898 \mathbb{R}^{\circ}$ 圈

\section{Resumo}

Este artigo tem o objectivo de realizar uma revisão não sistemática e narrativa da literatura de estudos que comparam a qualidade de vida de mulheres com cancro da mama submetidas à diferentes tipos de cirurgia. Ao analisar os estudos, verificou-se que estes abordavam diversos aspectos, agrupados em três dimensões: a) Metodologia adoptada, se referindo aos instrumentos utilizados, o intervalo de avaliação e o procedimento de recolha de dados; b) Variáveis sociodemográficas e psicoemocionais, que diz respeito à idade, escolaridade, estado civil, sexualidade e percepção da imagem corporal; e c) Variáveis clínicas, relacionado ao tipo de tratamento e ao estádio da doença. Os resultados levam-nos a reflectir sobre a importância de incluir a avaliação da qualidade de vida no processo de tratamento e no seguimento das doentes, independente do tipo de cirurgia a que as mulheres são submetidas.

Palavras-chave: Cancro Da Mama, Qualidade De Vida, Cirurgia, Revisão Narrativa.

\section{Abstract}

This article aims to conduct a non-systematic and narrative review of the literature of studies comparing the quality of life in women with breast cancer undergoing different types of surgery. Analyzing the studies showed several aspects, grouped into three dimensions: a) Methodology adopted, referring to the instruments, the interval of evaluation, and the procedure of data collection; b) Sociodemographic and psychoemotional variables, related to age, educational level, marital status, sexuality and perception of body image; and c) Clinical variables, related to the type of treatment and the stage of the disease. Results leads us to reflect on the importance of including the assessment of quality of life in the treatment process and follow-up of patients, regardless of the type of surgery that women are submitted.

Keywords: Breast Cancer, Quality of Life, Surgery, Narrative Review. 
Pág 36

2. Doutor em Psicologia, Professor Auxiliar da Faculdade de Psicologia e de Ciências da Educação da Universidade do Porto, Portugal.

\section{Resumen}

Este artículo tiene como objetivo realizar una revisión no sistemática y narrativa de la literatura de estudios que comparan la calidad de vida de mujeres con cáncer de mama sometidas a diferentes tipos de cirugía. Mediante el análisis de los estudios, se encontró diversos aspectos, agrupados en tres dimensiones: a) Metodología utilizada, se refiere a los instrumentos, el intervalo de evaluación, así como el procedimiento de recolección de datos; b) Variables socio-demográficas y psico-emocionales, relativas a la edad, la educación, el estado civil, la sexualidad y la percepción de la imagen corporal; y c) Variables clínicas, relacionadas con el tipo de tratamiento y lo estadio de la enfermedad. Los resultados nos llevan a reflexionar sobre la importancia de incluir la evaluación de la calidad de vida en el proceso de tratamiento y el seguimiento de los pacientes, sin importar el tipo de cirugía al que las mujeres son sometidas.

Palabras clave: Cáncer De Mama, Calidad De Vida, Cirugía, Revisión Narrativa.

\section{Tipos de tratamento cirúrgico}

Para a maioria das mulheres com cancro da mama, o tratamento inclui algum tipo de cirurgia. Nos casos em que há indicação de cirurgia, tanto a mastectomia, com ou sem reconstrução, ou a cirurgia conservadora da mama são muitas vezes as opções viáveis (Parker et al., 2007; Pockaj et al., 2009).

Relativamente ao tratamento, pode-se observar que as cirurgias estão cada vez mais presentes, de formas menos radicais do que no passado, sendo complementadas muitas vezes com a quimioterapia ou a radioterapia. Estima-se que um terço das mulheres com cancro da mama sejam submetidas à mastectomia (Boukerrou, Saal, Laurent, Barau, \& Clough et al., 2010; Lardi et al., 2013).

Durante muitas décadas a mastectomia imperava como procedimento cirúrgico para o tratamento do cancro da mama. Este tipo de cirurgia foi desenvolvido por William Halsted no final do século XIX, levando o nome do seu criador: mastectomia radical de Halsted. Este procedimento cirúrgico consistia na remoção total da mama e incluía os músculos peitorais, a pele e os gânglios linfáticos axilares. Esta modalidade cirúrgica teve bons resultados, aumentando em $40 \%$ a sobrevida da paciente após cinco anos, considerado um avanço para a época (Loukas et al., 2011). Entretanto, a mastectomia radical trazia consigo inúmeros efeitos secundários, o que motivou a necessidade de aperfeiçoar a técnica, com vista à maior preservação dos tecidos. De facto, com o passar do tempo, o radicalismo tradicional perdeu espaço, e novas técnicas foram introduzidas, na segunda metade do século XX, por Patey, Madden, entre outros especialistas, que popularizaram as mastectomias radicais modificadas. Patey introduziu a cirurgia associada à exérese do peitoral menor, enquanto Madden desenvolveu a técnica que preserva ambos os peitorais. Com a introdução destas novas técnicas os médicos começaram a comparar e avaliar os resultados entre a mastectomia radical e os procedimentos mais conservadores (Champaneria, Wong, Hill, \& Gupta, 2012; Cotlar, Dubose, \& Rose, 2003).

A crescente introdução da cirurgia conservadora levou ao desenvolvimento de vários ensaios clínicos, por exemplo o Milan-World Health Organization Veronesi et al., 1990), o European Organization for the Research and Treatment of Cancer (EORTC) 10801 (Van Dongen et al., 2000), dentre outros. A investigação veio a demonstrar que, 
Pág 37

A qualidade de vida relacionada com a saúde é considerada hoje, juntamente com a sobrevida livre de doença um dos parâmetros mais importantes para avaliar o impacto de um tratamento em pacientes com cancro, em dimensões que incluem e ultrapassam as questões meramente biológicas (Dorcaratto, Grande, Ramón, \& Pera, 2011). para doentes em estádios iniciais, a mastectomia e a cirurgia conservadora seguida de radioterapia tinham taxas de sobrevivências semelhantes. Após estes estudos, foi organizado o Consensus Development Conference (NHI Consensus Development Panel, 1991), tendo-se concluído que a cirurgia conservadora era preferível à mastectomia para mulheres de determinada condição clínica, possibilitando a preservação da mama.

Os avanços nas técnicas cirúrgicas continuaram e a reconstrução mamária após a mastectomia tornou-se popular no final do século XX, e evoluiu muito no que se refere à segurança e aplicabilidade da mesma. Tornou-se uma nova opção para os cirurgiões e oncologistas, e também para as mulheres, ao proporcionar benefícios a nível psicológico e de autoimagem (Malata, Mclntosh, \& Purushotham, 2000).

\section{Qualidade de vida}

No início dos anos 80, a qualidade de vida do doente passou a ser uma preocupação a ser atendida em oncologia, e o interesse por técnicas cirúrgicas mais conservadoras aumenta, de modo a contribuir fortemente para a melhoria da qualidade de vida do paciente. Qualidade de vida é um conceito complexo, abstrato e multidimensional, difícil de definir (Schlarmann, Metzing-Blau, \& Schnepp, 2008).

Segundo a Organização Mundial da Saúde (WHO, 1995), a qualidade de vida compreende aspectos objectivos e subjectivos, e inclui a funcionalidade, a competência cognitiva, a interacção do indivíduo com o meio e a percepção, por parte de indivíduos ou grupos, da satisfação das suas necessidades e daquilo que não lhes é recusado nas ocasiões propícias à sua realização e à sua felicidade. É um estado de completo bem-estar físico, mental e social e não meramente a ausência de doença.

Quando a qualidade de vida diz respeito à saúde, tem-se o conceito de "qualidade de vida relacionada com a saúde". Assim como o conceito de qualidade de vida, não existe um único conceito de qualidade de vida relacionada com a saúde. Um dos conceitos difundidos diz que "é a percepção dos doentes sobre as suas capacidades em quatro grandes dimensões: bem-estar físico e actividades quotidianas, bem-estar psicológico, relações sociais e sintomas. [...] É um conceito multidimensional, subjectivo e individual" (Pimentel, 2006, p.12). A qualidade de vida relacionada com a saúde é considerada hoje, juntamente com a sobrevida livre de doença, um dos parâmetros mais importantes para avaliar o impacto de um tratamento em pacientes com cancro, em dimensões que incluem e ultrapassam as questões meramente biológicas (Dorcaratto, Grande, Ramón, \& Pera, 2011).

Várias técnicas e instrumentos foram desenvolvidos para avaliar as funções do indivíduo, a situação de saúde e a qualidade de vida. Entre as medidas específicas para avaliar qualidade de vida em doentes oncológicos destacam-se: European Organization for Research and Treatment of Cancer Quality of Life Questionnaire (EORTC QLQ-C30) e Functional Assessment of Cancer Therapy (FACT). Entre as medidas mais usadas para avaliar qualidade de vida, em geral, destacam-se: Medical Outcomes Study 36-Item Short-Form Healty Survey (SF-36) e World Health Organization Quality of Life (WHOQOL-100 ou WHOQOL-bref). De modo geral, os questionários utilizados para mensurar a qualidade de vida abordam questões semelhantes para avaliar os aspectos como estado geral de saúde, função física, emocional, cognitiva e social, e alguns incluem também escalas de sintomas, como por exemplo náusea e dor (Pimentel, 2006). 
Nos últimos anos têm crescido o número de estudos sobre a qualidade de vida em pacientes com cancro, e a avaliação da mesma é cada vez mais frequente em ensaios clínicos, na avaliação por rotina dos doentes em tratamento ou no seguimento dos doentes após o tratamento. Uma consulta realizada na base de dados EBSCO, em Dezembro de 2016, com os termos "quality of life", "breast cancer" e "surgery" resultou em 4.331 publicações, constatando-se um número crescente de estudos publicados nos últimos anos (ver figura 1).

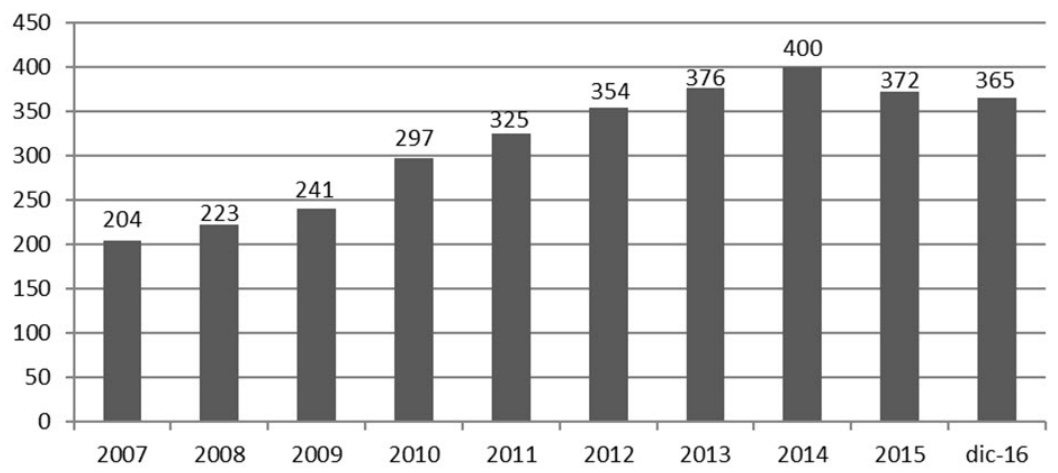

Figura 1. Publicações com os termos "quality of life", "breast cancer" e "surgery" (2007-2016).

Neste sentido, o objetivo deste artigo é realizar uma revisão não sistemática e narrativa da literatura de estudos sobre a qualidade de vida de mulheres com cancro da mama que comparam os tipos de cirurgia.

\section{Método}

A revisão narrativa baseou-se na pesquisa de estudos empíricos, realizada na base de dados EBSCO. Este tipo de revisão consiste numa revisão qualitativa, na qual o pesquisador procura sintetizar e resumir os resultados de informações publicadas anteriormente (Green, Johnson, \& Adams, 2006). Segundo Rother (2007), este tipo de revisão possibilita o autor fazer análises e interpretações críticas mais ampliadas permitindo uma melhor compreensão do "estado da arte" de um assunto específico. Não é uma condição para realização de revisões narrativas informar o método de busca de referências, nem os critérios adoptados para avaliação e seleção dos artigos, visto que a revisão narrativa é de natureza opinativa, ou seja, o próprio autor "decide quais as informações são mais relevantes, sem explicitar a forma como elas são obtidas" (Bernardo, Nobre, \& Jatene, 2004, p.104)

\section{Resultados e Discussão}

Ao analisar os estudos que avaliam e comparam a qualidade de vida em mulheres com cancro de mama submetidas à cirurgia, verificou-se que estes abordavam diversos aspectos, pelo que, optou-se por agrupá-los em três dimensões gerais: a) Metodologia adoptada; b) Variáveis sociodemográficas e psicoemocionais; e c) Variáveis clínicas (ver tabela 1$)$. 
Tabela 1. Dimensões e Aspectos Abordados nos Estudos.

\begin{tabular}{ll}
\hline \multicolumn{1}{c}{ Dimensões } & \multicolumn{1}{c}{ Aspectos } \\
\hline \multirow{2}{*}{ Metodologia adoptada } & - Instrumentos utilizados \\
& - Intervalo de avaliação (follow-up) \\
& - Procedimento de recolha de dados \\
\hline & - Idade \\
Variáveis sociodemográficas e & - Escolaridade \\
psicoemocionais & - Estado civil \\
& - Sexualidade \\
& - Percepção da imagem corporal \\
\hline \multirow{2}{*}{ Variáveis clínicas } & - Tipo de tratamento \\
& - Estádio da doença \\
\hline
\end{tabular}

\section{Metodologia adoptada}

Inicialmente, constatou-se que a primeira diferença que surge, ao analisar os estudos, diz respeito à ampla variedade de instrumentos utilizados para avaliar a qualidade de vida. Um dos principais questionários é o EORTC QLQ-C30, usado em combinação com o QLQ-BR23, módulo específico para o cancro da mama. Este questionário é o mais utilizado na Europa e largamente utilizado no resto do mundo (Han, Grothuesmann, Neises, Hille, \& Hillemanns, 2010; Shi et al., 2011). Outro instrumento amplamente utilizado é o FACT, muito similar ao QLQ-C30, e conta também com um módulo específico para o cancro da mama, o FACT-B ( tigadores optam por utilizar questionários genéricos, não específicos para doentes oncológicos, sendo um dos mais utilizados mundialmente o questionário SF-36 (Parker et al., 2007; Veiga et al., 2010).

Outro aspecto encontrado se refere ao padrão de tempo e intervalo de avaliação, ou seja, cada pesquisador determina em que momento avaliar a qualidade de vida, e por quantas vezes repetir a avaliação, no período do follow-up. Diversos estudos são desenvolvidos nos primeiros meses após a cirurgia, sendo o follow-up das investigações geralmente inferior a dois anos (Han et al., 2010; Parker et al., 2007; Shi et al., 2011; Veiga et al., 2010). São poucos os estudos com seguimento prolongado dos participantes (Arndt, Stegmaier, Ziegler, \& Brenner, 2008; Ganz et al., 2002).

Num estudo desenvolvido nos Estados Unidos com 258 mulheres, das quais 109 fizeram mastectomia com reconstrução, 45 mastectomia sem reconstrução e 104 cirurgia conservadora, avaliou-se a qualidade de vida em cinco momentos: no pré-operatório e após 1, 6, 12 e 24 meses da cirurgia (Parker et al., 2007). 0 estudo mostrou que os escores de qualidade de vida no pré-operatório das mulheres que fizeram mastectomia eram mais baixos do que as que fizeram cirurgia conservadora, sendo que com o passar do tempo, principalmente no último momento da avaliação os escores eram muito semelhantes.

Num outro estudo, realizado em Taiwan, que utilizou um método semelhante, participaram 173 mulheres que responderam os questionários em três momentos: no pré-operatório, e ao final do primeiro e do segundo ano de follow-up (Shi et al., 2011). Verificou-se que a qualidade de vida de todos os grupos melhorou com o passar do tempo, e o grupo submetido à cirurgia conservadora teve escores mais elevados. 
É importante compreender que a forma como as pessoas percepcionam a qualidade de vida muda ao longo do tempo e é modificada pela idade e experiências vividas (Calman, 1984). A qualidade de vida tende a melhorar com o passar do tempo, uma vez que os factores que influenciam a qualidade de vida tendem a diminuir seu impacto, e por isso considera-se relevante a aplicação dos questionários em diferentes momentos após a cirurgia, a fim de acompanhar as mudanças.
0 efeito do tempo na qualidade de vida também pode ser observado num estudo realizado por Arndt e colaboradores (2008) que verificaram que as diferenças na qualidade de vida global entre as mulheres submetidas à cirurgia conservadora ou mastectomia aumentavam gradualmente com o passar do tempo, e tornaram-se estatisticamente significativas somente na avaliação aos cinco anos após o diagnóstico, com melhores resultados para o grupo da cirurgia conservadora. Durante o follow-up a qualidade de vida global diminuiu para ambos os grupos, porém teve decréscimo mais acentuado nas mulheres submetidas à mastectomia. As mulheres que fizeram cirurgia conservadora apresentaram escores superiores em todos os períodos de avaliação.

É importante compreender que a forma como as pessoas percepcionam a qualidade de vida muda ao longo do tempo e é modificada pela idade e experiências vividas (Calman, 1984). A qualidade de vida tende a melhorar com o passar do tempo, uma vez que os factores que influenciam a qualidade de vida tendem a diminuir seu impacto, e por isso considera-se relevante a aplicação dos questionários em diferentes momentos após a cirurgia, a fim de acompanhar as mudanças. Dessa forma, alguns estudos sugerem que o potencial impacto positivo da cirurgia conservadora na adaptação da doente pode apenas ser evidente alguns meses ou anos após o diagnóstico e tratamento inicial (Pockaj et al., 2009).

De fato, um dos principais objectivos da avaliação da qualidade de vida é a possibilidade de comparar resultados entre grupos de doentes e modalidades de tratamentos (Pimentel, 2006). Quando os estudos que avaliam a qualidade de vida diferem no que diz respeito aos processos de avaliação, ou seja, nos instrumentos utilizados, como também nas repetições da avaliação durante o follow-up, torna a comparação de resultados entre estudos mais difícil.

Outra diferença entre os estudos encontrados na literatura é o procedimento utilizado pelos investigadores para a recolha de dados. Alguns autores optaram por enviar os questionários por correspondência ou por meios electrónicos às pacientes para serem auto-aplicados ( optaram por aplicar os questionários na consulta hospitalar de follow-up, geralmente com a presença do próprio investigador (He et al., 2012; Shi et al., 2011; Veiga et al., 2010).

Entende-se que os questionários respondidos à distância, por meios electrónicos ou por correspondência facilitam a inclusão de indivíduos no estudo, uma vez que os mesmos não precisam de se deslocar até o centro de investigação ou hospital. Por outro lado, questionários enviados por meios electrónicos ou por correspondência tornam a adesão ao estudo condicionada, quase que exclusivamente, pela própria iniciativa do paciente.

Além disso, os questionários respondidos à distância podem trazer consequências para os resultados da avaliação, uma vez que os pacientes podem sofrer influência das pessoas próximas ao responder os inquéritos, além de estarem susceptíveis a erros de interpretação das questões, uma vez que a ausência do investigador impediria o esclarecimento de possíveis dúvidas que o paciente possa ter ao responder o questionário. É importante ressaltar que muitos dos questionários de avaliação da qualidade de vida foram desenvolvidos para serem autopreenchidos, uma vez que a qualidade de vida individual depende tanto do indivíduo, que só o próprio é capaz de um juízo de valor sobre a sua qualidade de vida (Calman, 1984). 


\section{Variáveis Sociodemográficas e Psicoemocionais}

Factores como idade e estado civil podem afectar claramente o ajustamento psicossocial e os resultados da qualidade de vida (Aizer et al., 2013; Brunault et al., 2015; Ganz et al., 2002).

No que se refere à idade, um estudo mostrou que, em uma avaliação feita antes da cirurgia, as mulheres mais velhas mostraram-se menos depressivas e ansiosas, e com melhores resultados para a qualidade de vida global do que as mulheres mais jovens (Parker, 2007). Outro estudo indicou que as mulheres mais jovens submetidas à mastectomia tinham escores mais baixos no domínio psicológico do que as mulheres mais velhas, também submetidas à mastectomia. Além disso, este mesmo estudo comparou mulheres mastectomizadas com mulheres que fizeram cirurgia conservadora, e dentre as mulheres mais jovens dos dois grupos observou-se diferença significativa nos domínios da função física, dor e função social, sendo que as mulheres mastectomizadas tiveram escores mais baixos (Veiga et al., 2010).

A literatura mostra também que a idade da mulher no momento do diagnóstico tem influência na qualidade de vida. 0 diagnóstico de cancro da mama traz grande impacto na vida das mulheres jovens, uma vez que este grupo é mais vulnerável à ansiedade relacionada ao tempo de sobrevivência, e também com os efeitos estéticos que um tratamento cirúrgico pode trazer, além das implicações relacionadas ao trabalho, à vida conjugal e à maternidade, aspectos esses que geralmente são mais facilmente superados pelas mulheres mais velhas (Avis, Crawford, \& Manuel, 2005; Howard-Anderson, Ganz, Bower, \& Stanton, 2012).

Investigações que incluíram a escolaridade das mulheres em suas avaliações mostraram que as mulheres com maior escolaridade, que foram submetidas à mastectomia, estavam mais satisfeitas com a sua imagem corporal após a cirurgia do que as com escolaridade mais baixa (Parker et al., 2007; Veiga et al., 2010). Em outro estudo, o nível de escolaridade também foi considerado um factor positivo sobre a qualidade de vida. Mulheres com maior escolaridade apresentaram escores mais altos de função física, função emocional, menos dor e poucos sintomas na mama, em relação a muIheres com baixa escolaridade (King, Kenny, Shiell, Hall, \& Boyages, 2000).

Segundo Veiga e colaboradores (2010), a relação entre a escolaridade e a qualidade de vida pode estar atribuída ao facto de que as mulheres com escolaridade mais baixa realizam trabalhos que exigem maiores esforços físicos, logo são mais prejudicadas no trabalho por conta do tratamento. Por outro lado, King e colaboradores (2000) procuram justificativa no facto de que as mulheres com maior escolaridade têm mais acesso à informação sobre o autocuidado e estão mais preparadas para lidar com possíveis sintomas, como, por exemplo, a dor.

Relativamente ao estado civil, um estudo apontou que mulheres casadas apresentam melhores escores de qualidade de vida global do que mulheres solteiras, entretanto o impacto da mastectomia sobre a imagem corporal é maior em mulheres casadas do que em mulheres solteiras (King et al., 2000). Outro estudo mostrou que as mulheres casadas têm melhores resultados referentes aos aspectos da sexualidade, que engloba o desejo e o prazer sexual (Parker et al., 2007).

Ainda com relação à sexualidade, um estudo desenvolvido na China não encontrou diferenças entre as mulheres que fizeram mastectomia e as que fizeram cirurgia conservadora (He et al., 2012), facto que os próprios autores atribuíram à cultura, 
Pág 42

Na mulher que passa por uma experiência cirúrgica nas mamas, a imagem corporal pode ser influenciada por múltiplas variáveis como, por exemplo, o valor atribuído às mamas, a autoimagem pré e pós operatória, a relação com o parceiro, dentre outros factores que são muito pessoais, e variam de mulher para mulher. uma vez que as mulheres chinesas são relutantes para falar da sua própria sexualidade e expressar seus reais sentimentos sobre o sexo, o que pode ter levado à ausência de diferenças entre os grupos.

No que se refere à imagem corporal, percebe-se que esta dimensão também não apresenta consenso entre os investigadores. Diversos estudos relatam que a mastectomia influencia na percepção negativa da imagem corporal, em comparação com a cirurgia conservadora (Arndt et al., 2008; Markopoulos et al., 2009; Shi et al., 2011) sendo um resultado previsível diante da retirada da mama. Há ainda autores que afirmam que as mulheres que fizeram cirurgia conservadora apresentaram uma melhor percepção da imagem corporal do que aquelas que fizeram a reconstrução imediata (Han et al., 2010), ou há aqueles que não encontraram diferenças entre esses grupos (Nano et al., 2005). Também há investigadores que mostraram que o tipo de cirurgia realizada não interfere na imagem corporal, seja ela conservadora, mastectomia com reconstrução ou mastectomia sem reconstrução (Poulsen, Graversen, Beckmann \& Blichert-Toft, 1997).

Estas divergências podem ser entendidas quando consideramos que a imagem corporal pode ser definida como a imagem mental de um corpo, idealizada, uma atitude sobre a autoaparência física e estado de saúde, o funcionamento normal e a sexualidade do próprio corpo (Fobair et al., 2006). Na mulher que passa por uma experiência cirúrgica nas mamas, a imagem corporal pode ser influenciada por múltiplas variáveis como, por exemplo, o valor atribuído às mamas, a autoimagem pré e pós operatória, a relação com o parceiro, dentre outros factores que são muito pessoais, e variam de mulher para mulher.

\section{Variáveis Clínicas}

0 tratamento das mulheres que foram submetidas a alguma cirurgia é muitas vezes complementado com radioterapia ou quimioterapia nos meses que se seguem à cirurgia. Associados à radioterapia e quimioterapia estão efeitos secundários diversos que podem influenciar negativamente a qualidade de vida da mulher (Palazzi et al. 2006).

A maioria das mulheres que realizam cirurgia conservadora tem seu tratamento complementado com a radioterapia. Neste sentido, alguns autores sugerem que a ausência de diferenças na qualidade de vida entre a mastectomia e a cirurgia conservadora, nos primeiros meses após a cirurgia, pode ser parcialmente explicada pela utilização da radioterapia imediatamente após a cirurgia conservadora ( al., 2012).

Um estudo mostrou que durante o tratamento com radioterapia, a qualidade de vida, as funções sociais e sexuais de pacientes submetidas à cirurgia conservadora sofreram diminuição de pontuação, o que foi revertido seis meses após, com o término da radioterapia (Dow \& Lafferty, 2000). Outro estudo comparou mulheres que fizeram radioterapia e mulheres que não fizeram radioterapia após cirurgia conservadora e indicou que, quem fez radioterapia teve um decréscimo na qualidade de vida somente no período do tratamento radioterápico, o que foi revertido imediatamente após o término do tratamento, igualando a qualidade de vida com o grupo que não fez radioterapia (Williams, Kunkler, King \& Jack, 2011). Desta forma, a radioterapia parece ter pouca influência na qualidade de vida global a longo prazo, mas muita influência a curto prazo (He et al., 2012). 
Pág 43

Ressalta-se a importância de considerar o estádio da doença, e também se as pacientes fizeram radioterapia ou quimioterapia nos estudos que comparam diferentes técnicas cirúrgicas, uma vez que estes aspectos têm grandes impactos na vida da mulher e podem influenciar os resultados da qualidade de vida.
Enquanto a radioterapia é mais frequentemente incluída em estudos sobre a qualidade de vida, são poucos os estudos que avaliam a influência da quimioterapia na qualidade de vida, como tratamento adjuvante à cirurgia. Porém, avaliar a sua influência é importante, uma vez que os efeitos secundários da mesma podem ter efeito negativo na qualidade de vida em vários aspectos como, por exemplo, nos aspectos sexuais e de imagem corporal (Schover et al., 1995).

Um estudo avaliou a qualidade de vida após cirurgia em quatro grupos de pacientes: a) mastectomia e quimioterapia, b) mastectomia sem quimioterapia, c) cirurgia conservadora e quimioterapia e d) cirurgia conservadora sem quimioterapia (Ganz et al., 2004). Os questionários foram respondidos três semanas após o fim do tratamento, seja ele apenas cirurgia ou cirurgia e quimioterapia. As pacientes que foram tratadas apenas com a cirurgia conservadora tiveram escores superiores na função física do que quem foi submetida apenas a mastectomia. Os escores para a função física foram melhores para as pacientes que apenas realizaram a cirurgia e não receberam quimioterapia após. Os investigadores não encontraram diferenças nas funções emocionais entre os grupos. De maneira geral, os escores de qualidade de vida foram mais baixos para as mulheres que foram submetidas à mastectomia complementada com a quimioterapia.

Por fim, percebe-se que as investigações não são homogéneas quanto à inclusão dos participantes, relativamente ao estádio da doença, o que dificulta a comparação de resultados. Muitos estudos foram realizados com mulheres em estádio I e II ( et al., 2012; Parker et al., 2007), entretanto há estudos que consideram as mulheres em estádio I, II, III (Han et al., 2010; Shi et al., 2011) e ainda estádio IV (Heneghan et al., 2011). Há também pesquisadores que não informaram o estádio das pacientes (Veiga et al, 2010).

Ressalta-se a importância de considerar o estádio da doença, e também se as pacientes fizeram radioterapia ou quimioterapia nos estudos que comparam diferentes técnicas cirúrgicas, uma vez que estes aspectos têm grandes impactos na vida da mulher e podem influenciar os resultados da qualidade de vida.

\section{Considerações Finais}

São inúmeros os estudos que avaliam a qualidade de vida de mulheres com cancro da mama que foram submetidas à cirurgia. Neste sentido, este estudo teve como objetivo fazer uma revisão narrativa da literatura sobre estes estudos. Foram identificados vários aspectos que foram agrupados em três dimensões: a) Metodologia adoptada, se referindo aos instrumentos utilizados, o intervalo de avaliação, e o procedimento de recolha de dados; b) Variáveis sociodemográficas e psicoemocionais, que diz respeito à idade, escolaridade, estado civil, sexualidade e percepção da imagem corporal; e c) Variáveis clínicas, relacionado ao tipo de tratamento e ao estádio da doença.

Os estudos que comparam a qualidade de vida em função da cirurgia são heterogéneos em termos metodológicos e relativamente às variáveis abordadas, levando a resultados diversos. Esta heterogeneidade entre os estudos pode ser justificada pelas variáveis que vão além das variáveis contidas nos questionários de qualidade de vida. Estudos que não consideram idade, estado civil, co-morbidades anteriores à cirurgia, climatério, radioterapia, quimioterapia, estádio da doença, questões inerentes à cultura, dentre outros, podem ter resultados que não serão fácil e correctamente compreendidos. 
A inclusão da avaliação da qualidade de vida nas pesquisas científicas sobre o cancro de mama é de extrema relevância, uma vez que esta avaliação pode guiar os profissionais de saúde à adopção de abordagens terapêuticas, bem como em orientações à paciente, desde o momento do diagnóstico da doença, com o intuito de minimizar ou contornar problemas, favorecendo a recuperação física e emocional da mulher. Por isso, destaca-se que a decisão sobre qual o melhor tipo de cirurgia deve ser sempre individualizada e considerar diversos factores relacionados à situação clínica da doente (por exemplo o estadiamento do tumor), e para além disso também deve considerar a qualidade de vida possibilitada pela cirurgia.

Ao conhecer o problema e entender os determinantes da qualidade de vida, os profissionais da saúde envolvidos no cuidado à mulher estarão mais capacitados para actuar com intervenções eficazes no tratamento, visando uma melhor qualidade de vida à paciente.

\section{Referências}

Aizer, A.A., Chen, M-H., McCarthy, E.P., Mendu, M.L., Koo, S., Wilhite, T.J., ... Nguyen, P.L. (2013). Marital status and survival in patients with cancer. Journal of Clinical Oncology, 31(31), 3869-3876. http://dx.doi.org/ 10.1200/jco.2013.49.6489

Arndt, V., Stegmaier, C., Ziegler, H., \& Brenner, H. (2008). Quality of life over 5 years in women with breast cancer after breast-conserving therapy versus mastectomy: a population-based study. Journal of Cancer Research and Clinical Oncology, 134(12), 1311-1318. http://dx.doi.org/ 10.1007/s00432-008-0418-y

Avis, N., Crawford, S., \& Manuel, J. (2005). Quality of life among younger women with breast cancer. Journal of Clinical Oncology, 23(15), 3322-3330. http://dx.doi.org/ 10.1200/jco.2005.05.130

Bernardo, W.M., Nobre, M.R.C., \& Jatene, F.B. (2004). A prática clínica baseada em evidências: parte II - buscando as evidências em fontes de informação. Revista da Associação Médica Brasileira, 50(1), 104-108. http://dx.doi.org/ 10.1590/S010442302004000100045

Boukerrou, M., Saal, J.D., Laurent, T., Barau, G., \& Clough, K. (2010). Mastectomie totale avec conservation de la plaque aréolo-mammelonnaire: état des lieux. Gynecologie Obstetrique \& Fertilite, 38(10), 600-606. http://dx.doi.org/ 10.1016/j. gyobfe. 2010.08 .013

Brunault, P., Champagne, A-L., Huguet, G., Suzanne, I., Senon, J., Body, G., ... Camus, V. (2015). Major depressive disorder, personality disorders, and coping strategies are independent risk factors for lower quality of life in non?metastatic breast cancer patients. Psycho? 0 ncology, 25, 513-520. http://dx.doi.org/ $10.1002 /$ pon. 3947

Calman, K. (1984). Quality of life in cancer patients: an hypothesis. Journal of Medical Ethics, 10(3), 124-127. http://dx.doi.org/ 10.1136/jme.10.3.124

Champaneria, M., Wong, W., Hill, M., \& Gupta, S. (2012). The evolution of breast reconstruction: a historical perspective. World Journal of Surgery, 36(4), 730-742. http://dx.doi.org/ 10.1007/s00268-012-1450-2

Cotlar, A., Dubose, J., \& Rose, D. (2003). History of surgery for breast cancer: radical to the sublime. Current Surgery, 60(3), 329-337. http://dx.doi.org/ 10.1016/s01497944(02)00777-8

Dorcaratto, D., Grande, L., Ramón, J., \& Pera, M. (2011). Quality of life of patients with cancer of the oesophagus and stomach. Cirugía Española, 89(10), 635-644. http://dx.doi.org/ 10.1016/j.ciresp.2011.07.006 
Dow, K., \& Lafferty, P. (2000). Quality of life, survivorship, and psychosocial adjustment of young women with breast cancer after breast-conserving surgery and radiation therapy. Oncology Nursing Forum, 27(10), 1555-1564. https://www. ncbi.nlm.nih.gov/pubmed/11103374

Ferlay, J., Soerjomataram, I., Ervik, M., Dikshit, R., Eser, S., Mathers, C., ...Bray, F. (2013). GLOBOCAN 2012, Cancer incidence and mortality worldwide: IARC CancerBase International Agency for Research on Cancer 11. http://globocan.iarc.fr (Acedido em 31/10/2016).

Fobair, P., Stewart, S., Chang, S., D'Onofrio, C., Banks, P., \& Bloom, J. (2006). Body image and sexual problems in young women with breast cancer. Psycho-Oncology, 15(7), 579-594. http://dx.doi.org/ 10.1002/pon.991

Ganz, P., Desmond, K., Leedham, B., Rowland, J., Meyerowitz, B., \& Belin, T. (2002). Quality of life in long-term, disease-free survivors of breast cancer: a follow-up study. Journal of the National Cancer Institute, 94(1), 39-49. https://www.ncbi. nlm.nih.gov/pubmed/11773281

Ganz, P., Kwan, L., Stanton, A., Krupnick, J., Rowland, J., Meyerowitz, B., ...Belin, T. (2004). Quality of life at the end of primary treatment of breast cancer: First results from the moving beyond cancer randomized trial. Journal of the National Cancer Institute, 96(5), 376-387. http://dx.doi.org/ 10.1093/jnci/djh060

Green, B., Johnson, C., \& Adams, A. (2006). Writing narrative literature reviews for peer-reviewed journals: secrets of the trade. Journal of Chiropractic Medicine, 5, 101-117. http://dx.doi.org/org/10.1016/S0899-3467(07)60142-6

Han, J., Grothuesmann, D., Neises, M., Hille, U., \& Hillemanns, P. (2010). Quality of life and satisfaction after breast cancer operation. Archives Of Gynecology And Obstetrics, 282(1), 75-82. http://dx.doi.org/ 10.1007/s00404-009-1302-y

He, Z., Tong, Q., Wu, S., Li, F., Lin, H., \& Guan, X. (2012). A comparison of quality of life and satisfaction of women with early-stage breast cancer treated with breast conserving therapy vs. mastectomy in southern China. Supportive Care in Cancer, 20(10), 2441-2449. http://dx.doi.org/ 10.1007/s00520-011-1364-9

Heneghan, H., Prichard, R., Lyons, R., Regan, P., Kelly, J., Malone, C... Kerin, M. (2011). Quality of life after immediate breast reconstruction and skin-sparing mastectomy - a comparison with patients undergoing breast conserving surgery. European Journal of Surgical Oncology, 37(11), 937-943. http://dx.doi.org/ 10.1016/j. ejso.2011.08.126

Howard-Anderson, J., Ganz, P., Bower, J., \& Stanton, A. (2012). Quality of life, fertility concerns, and behavioral health outcomes in younger breast cancer survivors: a systematic review. Journal of the National Cancer Institute, 104(5), 386-405. http://dx.doi.org/ 10.1093/jnci/djr541

King, M., Kenny, P., Shiell, A., Hall, J., \& Boyages, J. (2000). Quality of life three months and one year after first treatment for early stage breast cancer: influence of treatment and patient characteristics. Quality of Life Research, 9(7), 789-800. https://www.ncbi.nlm.nih.gov/pubmed/11297021

Lardi, A., Myrick, M., Haug, M., Schaefer, D., Bitzer, J., Simmen, U., \& Güth, U. (2013). The option of delayed reconstructive surgery following mastectomy for invasive breast cancer: why do so few patients embrace this offer? European Journal Of Surgical Oncology, 39(1), 36-43. http://dx.doi.org/ 10.1016/j.ejso.2012.08.010

Loukas, M., Tubbs, R., Mirzayan, N., Shirak, M., Steinberg, A., \& Shoja, M. (2011). The history of mastectomy. The American Surgeon, 77(5), 566-571. https://www. ncbi.nlm.nih.gov/pubmed/21679589

Malata, C., McIntosh, S., \& Purushotham, A. (2000). Immediate breast reconstruction after mastectomy for cancer. British Journal of Surgery, 87(11), 1455-1472. http://dx.doi.org/ 10.1046/j.1365-2168.2000.01593.x 
Markopoulos, C., Tsaroucha, A., Kouskos, E., Mantas, D., Antonopoulou, Z., \& Karvelis, S. (2009). Impact of breast cancer surgery on the self-esteem and sexual life of female patients. Journal of International Medical Research, 37 (1), 182-188. http://dx.doi.org/ 10.1177/147323000903700122

Nano, M., Gill, P., Kollias, J., Bochner, M., Malycha, P., \& Winefield, H. (2005). Psychological impact and cosmetic outcome of surgical breast cancer strategies. ANZ Journal of Surgery, 75(11), 940-947. http://dx.doi.org/ 10.1111/j. 1445-2197.2005.03517.x

NHI Consensus Development Panel. (1991). Treatment of early-stage breast cancer: NHI Consensus Conference. The Journal of the American Medical Association, 265, 391-395. https://consensus.nih.gov/1990/1990earlystagebreastcancer081 html.htm

Palazzi, M., Tomatis, S., Valli, M., Guzzetti, R., Tonoli, S., Bertoni, F., ... Cafaro, I. (2006). Impact of radiotherapy technique on the outcome of early breast cancer treated with conservative surgery: A multicenter observational study on 1,176 patients. International Journal Of Radiation Oncology, Biology, Physics, 65(5), 1361-1367. http://dx.doi.org/ 10.1016/j.ijrobp.2006.03.023

Parker, P., Youssef, A., Walker, S., Basen-Engquist, K., Cohen, L., Gritz, E.,...Robb, G. (2007). Short-term and long-term psychosocial adjustment and quality of life in women undergoing different surgical procedures for breast cancer. Annals of Surgical Oncology, 14(11), 3078-3089. http://dx.doi.org/ 10.1245/s10434-0079413-9

Pimentel, F. (2006). Qualidade de vida e oncologia. Coimbra: Almedina. http://journalofagingandinnovation.org/volume2-edicao3-julho2013/qualidade-de-vida-em-oncologia/

Pockaj, B., Degnim, A., Boughey, J., Gray, R., McLaughlin, S., Dueck, A., ... Sloan, J. (2009). Quality of life after breast cancer surgery: What have we learned and where should we go next? Journal of Surgical Oncology, 99(7), 447-455. http:// dx.doi.org/ 10.1002/jso.21151

Poulsen, B., Graversen, H., Beckmann, J., \& Blichert-Toft, M. (1997). A comparative study of post-operative psychosocial function in women with primary operable breast cancer randomized to breast conservation therapy or mastectomy. European Journal of Surgical Oncology 23(4), 327-334. http://dx.doi.org/ 10.1016/ S0748-7983(97)90804-0

Rother, E. (2007). Revisão sistemática X revisão narrativa. Acta Paulista de Enfermagem, 20(2), v-vi. http://dx.doi.org/10.1590/S0103-21002007000200001

Schlarmann, J., Metzing-Blau, S., \& Schnepp, W. (2008). The use of health-related quality of life (HRQOL) in children and adolescents as an outcome criterion to evaluate family oriented support for young carers in Germany: an integrative review of the literature. BMC Public Health, 8(414), 1-10. http://dx.doi.org/ 10.1186/1471-2458-8-414

Schover, L., Yetman, R., Tuason, L., Meisler, E., Esselstyn, C., Hermann, R., ... Dowden, R. (1995). Partial mastectomy and breast reconstruction. A comparison of their effects on psychosocial adjustment, body image, and sexuality. Cancer, 75(1), 54-64. http://dx.doi.org/ 10.1002/1097-0142(19950101)75:1<54::AID-CNCR2820750111> 3.0.CO;2-1

Shi, H., Uen, Y., Yen, L., Culbertson, R., Juan, C., \& Hou, M. (2011). Two-year quality of life after breast cancer surgery: a comparison of three surgical procedures. European Journal of Surgical Oncology, 37(8), 695-702. http://dx.doi.org/ 10.1016/j.ejso. 2011.05 .008 
Tao, Z., Shi, A., Lu, C., Song, T., Zhang, Z., \& Zhao, J. (2015). Breast cancer: epidemiology and etiology. Cell Biochemistry and Biophysics, 72(2), 333-338. http://dx.doi. org/ 10.1007/s12013-014-0459-6

Van Dongen, J., Voogd, A., Fentiman, I., Legrand, C., Sylvester, R., Tong, D., ...Bartelink, H. (2000). Long-term results of a randomized trial comparing breast-conserving therapy with mastectomy: European Organization for Research and Treatment of Cancer 10801 Trial. Journal of the National Cancer Institute, 92(14), 11431150. https://www.ncbi.nlm.nih.gov/pubmed/10904087

Veiga, D., Campos, F., Ribeiro, L., Archangelo Jr, I., Filho, J., Juliano, Y., ... Ferreira, L. (2010). Mastectomy versus conservative surgical treatment: the impact on the quality of life of women with breast cancer. Revista Brasileira de Saude Materno Infantil, 10(1), 51-57. http://dx.doi.org/ 10.1590/S1519-38292010000100005

Veronesi, U., Salvadori, B., Luini, A., Banfi, A., Zucali, R., Del Vecchio, M., ...Farante, G. (1990). Conservative treatment of early breast cancer. Long-term results of 1232 cases treated with quadrantectomy, axillary dissection, and radiotherapy. Annals of Surgery, 211(3), 250-259. https://www.ncbi.nlm.nih.gov/pmc/articles/PMC1358428/

World Health Organization (WHO). (1995). The World Health Organization quality of life assessment (WHOQOL): Position paper from the World Health Organization. Social Science \& Medicine, 41(10), 1403-1409. http://dx.doi.org/ 10.1016/02779536(95)00112-K

Williams, L., Kunkler, I., King, C., \& Jack, W. (2011). A randomised controlled trial of post-operative radiotherapy following breast-conserving surgery in a minimum-risk population. Quality of life at 5 years in the PRIME trial. Health Technology Assessment, 15(12), 1-64. http://dx.doi.org/ 10.3310/hta15120 